\title{
Morphological Alterations and NO-Synthase Expression in the Heart after Continuous Light Exposure of Rats
}

\author{
L. PAULIS ${ }^{1,2}$, R. VAŽAN ${ }^{3,4}$, F. ŠIMKO ${ }^{1}$, O. PECHÁŇOVÁ ${ }^{2,5}, J_{\text {J STYK }}^{4}$, \\ P. BABÁL ${ }^{6}$, P. JANEGA ${ }^{2,6}$
}

${ }^{1}$ Institute of Pathophysiology, School of Medicine, Comenius University, Bratislava, Slovak Republic, ${ }^{2}$ Institute of Normal and Pathological Physiology, Slovak Academy of Sciences, Bratislava, Slovak Republic, ${ }^{3}$ Department of Physiology, School of Medicine, Comenius University, Bratislava, Slovak Republic, ${ }^{4}$ Institute for Heart Research, Slovak Academy of Sciences, Bratislava, Slovak Republic, ${ }^{5}$ Institute of Physiology and Centre for Cardiovascular Research, Academy of Sciences of the Czech Republic, Prague, Czech Republic, ${ }^{6}$ Department of Pathology, School of Medicine, Comenius University, Bratislava, Slovak Republic

Received July 4, 2007

Accepted August 28, 2007

On-line available September 5, 2007

\begin{abstract}
Summary
Although exposure to continuous light is associated with hypertension and modulates the outcome of ischemiareperfusion injury, less attention has been paid to its effects on cardiac morphology. We investigated whether 4-week exposure of experimental rats to continuous $24 \mathrm{~h} /$ day light can modify cardiac morphology, with focus on heart weight, fibrosis and collagen I/III ratio in correlation with NO-synthase expression. Two groups of male adult Wistar rats were studied: controls exposed to normal light/dark cycle (12 h/day light, $12 \mathrm{~h} /$ day dark) and rats exposed to continuous light. After 4 weeks of treatment the absolute and the relative heart weights were determined and myocardial fibrosis and collagen type I/III ratio were evaluated using picrosirius red staining. Endothelial and inducible NO-synthase expression was detected immunohistochemically. The exposure of rats to continuous light resulted in an increase of body weight with proportionally increased heart weight. Myocardial fibrosis remained unaffected but collagen I/III ratio increased. Neither endothelial nor inducible NO-synthase expression was altered in light-exposed rats. We conclude that the loss of structural homogeneity of the myocardium in favor of collagen type I might increase myocardial stiffness and contribute to functional alterations after continuous light exposure.
\end{abstract}

\section{Key words}

Myocardium $\bullet$ Melatonin $\bullet$ Fibrosis Collagen I/III $\bullet$ Nitric oxide synthase

\section{Introduction}

Melatonin, the product of the pineal gland, is involved in the regulation of many organs, including cardiovascular system (Važan et al. 2004). Moreover, decreased melatonin levels were reported in various pathological conditions including ischemic heart disease (Brugger et al. 1995) or hypertension with non-dipper pattern (Jonas et al. 2003).

Deficit of melatonin may be experimentally 
induced by surgical pinealectomy (removal of epiphysis), which decreases day- and night-time melatonin levels (Brown et al. 1991). Pinealectomy is associated with temporarily increased blood pressure (Zanoboni and Zanoboni-Muciaccia 1967) and myocardial fibrosis (Mizrak et al. 2004). Another approach to decrease melatonin levels is the long-term exposure to continuous light, which abolishes nocturnal rise of melatonin serum levels (Brown et al. 1991). Our recent reports have shown that exposure of rats to continuous light modified the susceptibility of rat hearts to ischemia-reperfusion and attenuated cardiac NO-synthase activity (Paulis et al. 2006). Since NO was shown to inhibit proliferation (Garg and Hassid 1989) and fibrosis (Kolpakov et al. 1995) in the smooth muscle cells culture, continuous light may induce morphological changes in the myocardium, which could participate in the modulation of cardiac response to ischemia-reperfusion injury.

In the present study, we investigated whether four-week exposure of experimental rats to continuous (24 h/day) light can modify heart weight, fibrosis, collagen type I/III ratio and NO-synthase expression.

\section{Materials and Methods}

\section{Experimental animals}

The experiments were performed on adult male Wistar rats (body weight 250-320 g), in accordance with the Guide for the Care and Use of Laboratory Animals published by the US National Institutes of Health (NIH publication No. 85-23, revised 1996). Rats were housed under standard laboratory conditions and fed with standard pellet diet and tap water ad libitum. The room for rats was windowless with automatic regulation of light/dark cycle. Controls $(n=6)$ were housed under standard $12 \mathrm{~h}$ /day light, $12 \mathrm{~h} /$ day dark cycle and animals exposed to continuous light $(n=6)$ under $24 \mathrm{~h} /$ day light (450 lux) for 4 weeks. After the animals were sacrificed the hearts were removed weighted and the relative heart weight was calculated (heart weight/body weight).

\section{Myocardial fibrosis}

Samples from the left ventricle were fixed in $4 \%$ formaldehyde (Sigma Chemie, Germany) for $24 \mathrm{~h}$, embedded in paraffin, cut in $5 \mu \mathrm{m}$ slices and stained routinely with hematoxylin and eosin. Modified picrosirius red staining technique (Dolber and Spach 1993) was used to determine myocardial fibrosis. After deparaffinisation in xylene and rehydration in distilled water, the slides were incubated for $5 \mathrm{~min}$ in $0.2 \%$ phosphomolybdenic acid (Sigma Chemie, Germany) and stained with $0.1 \%$ sirius red F3BA in saturated picric acid solution (Sigma Chemie, Germany) for $90 \mathrm{~min}$. Finally slides were washed $2 \mathrm{~min}$ in $0.01 \mathrm{~mol} / \mathrm{l}$ hydrochloric acid. Sections were evaluated by microscopy in polarized light at the 40x magnification and myocardial fibrosis was measured by histomorphometry in 10 microscopic fields using the ImageJ morphometric software v.1.33 (National Institutes of Health, USA).

\section{Collagen types I/III ratio}

The slices stained with picrosirius red were used for collagen types I/III ratio analysis. The areas rich for collagen type I (red color in polarized light) and collagen type III (green color in polarized light) were analyzed by digital color subtraction using ImageJ software and collagen type I/III ratio was calculated.

\section{NO-synthase expression}

Nitric oxide synthase expression was analyzed by immunohistochemistry using mouse monoclonal antibodies directed against endothelial and inducible NOsynthase (BD Transduction Laboratories, USA). Deparaffinized sections were rehydrated in phosphatebuffered physiological salt solution (PBS) with $0.5 \%$ bovine serum albumin, $0.05 \% \mathrm{NaN}_{3}$ and $0.005 \%$ Tween 80, $\mathrm{pH}$ 7.2. After $1 \mathrm{~h}$ incubation with primary antibodies (1:100) at room temperature and three rinsing steps of $5 \mathrm{~min}$ each in PBS, sections were incubated for $15 \mathrm{~min}$ with the streptavidin-peroxidase complex (Dako, Carpinteria CA, USA). Peroxidase activity was visualized with diaminobenzidine (Zymed, San Francisco CA, USA) as substrate and sections were counterstained with hematoxyllin.

\section{Statistics}

Data are presented as means \pm S.E.M. Results were considered significant when $\mathrm{p}<0.05$. Two-tailed unpaired Student t-test was used. Normality of distribution was tested according to Kolmogorov and Smirnov and the difference in standard deviations was tested by Barlett's test.

\section{Results}

Heart weight, body weight and relative heart weight

Heart weight of control rats was $0.89 \pm 0.02 \mathrm{~g}$, the exposure of rats to continuous light increased the heart 
A

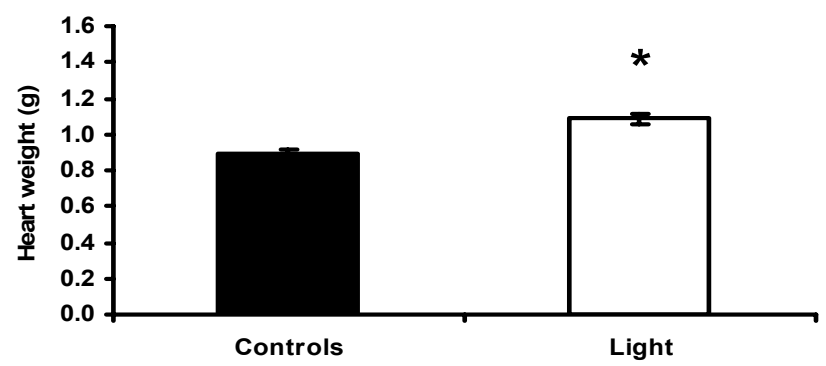

B

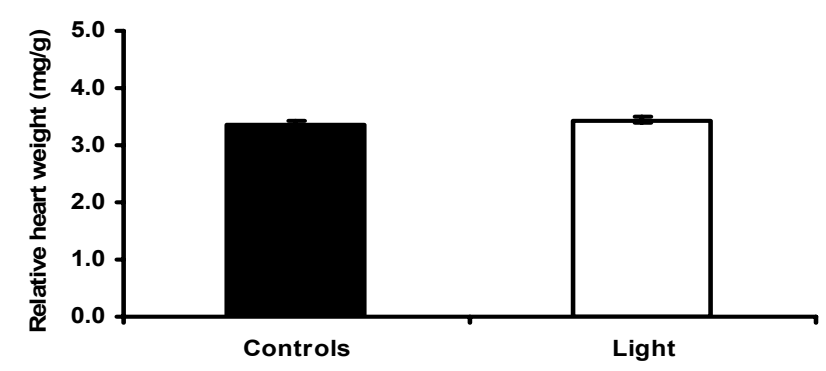

Fig. 1. Heart weight (A) and relative heart weight (heart weight/body weight) (B) in control group (control) and rats exposed for 4 weeks to continuous $24 \mathrm{~h} /$ day light (light). Data are means \pm SEM. $* p<0.05$ Student t-test.

weight by $21 \%(\mathrm{p}<0.05)$ (Fig. 1A). Body weight of control rats was $265 \pm 4.3 \mathrm{~g}$, the exposure of rats to continuous light increased body weight by $18 \%$ $(\mathrm{p}<0.05)$. The relative heart weight (heart weight/body weight) was $3.36 \pm 0.06 \mathrm{~g} / \mathrm{kg}$ in controls and the exposure of rats to continuous light had no significant effect on the relative heart weight (Fig. 1B).

\section{Myocardial fibrosis}

The total level of myocardial fibrosis of control rats was $2.26 \pm 0.23 \%$, the exposure of rats to continuous light had no significant effect on myocardial fibrosis (Fig. 2A).

\section{Collagen types I/III ratio}

The collagen type I/III ratio of control rats was $3.39 \pm 018$, the exposure of rats to continuous light increased the type I/III ratio by $37 \%(\mathrm{p}<0.001)$ (Fig. $2 \mathrm{~B})$.

\section{NO-synthase expression}

Both types (endothelial and inducible) of NOsynthase were present in myocardial cells showing cytoplasmatic granular positivity. One third of cardiomyocytes did not show any NO-synthase positivity. No differences between experimental groups in the localization and expression of either isoform of NOsynthase were observed.
A

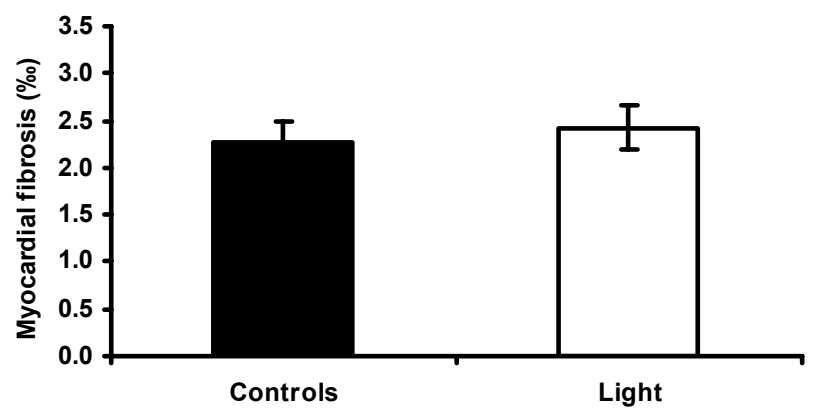

B

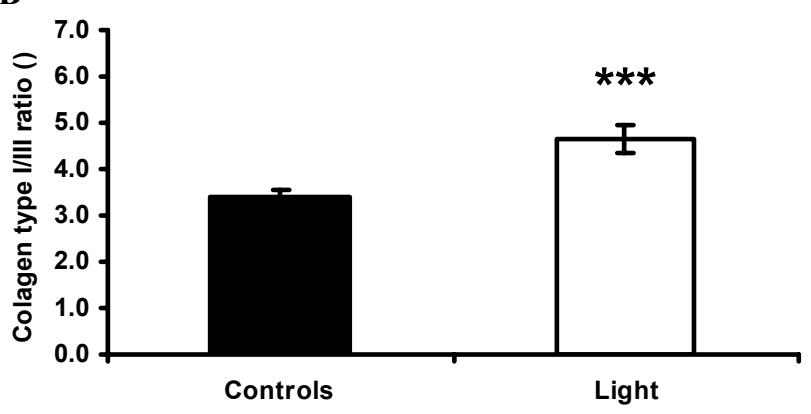

Fig. 2. Myocardial fibrosis (A) and collagen type I/III ratio (B) in control group (control) and rats exposed for 4 weeks to continuous $24 \mathrm{~h} /$ day light (light). Data are means \pm SEM. $* * * \mathrm{p}<0.001$ Student t-test.

\section{Discussion}

We investigated the effect of 4-week continuous light exposure of experimental rats on morphological changes and NO-synthase expression in the myocardium. The light-exposure increased heart weight and collagen type I/III ratio, but the relative heart weight, level of fibrosis and NO-synthase expression remained unaffected.

The exposure to continuous light, which abolishes the nocturnal rise of melatonin levels (Brown et al. 1991), represents a well-established model of "functional pinealectomy" (Barrett et al. 2000, Delibas et al. 2002, Briaud et al. 2004). Light exposure suppressed circadian rhythms of blood pressure and heart rate already after 3 weeks (Witte et al. 1998) and increased systolic and diastolic blood pressure (Briaud et al. 2004). Recently we have reported that the exposure of rats to continuous light has altered cardiac susceptibility to ischemia-reperfusion and has decreased cardiac NO synthase activity (Paulis et al. 2006). However, limited data are available on the effects of continuous light on morphological alterations in the heart, potentially accompanying the functional changes.

The "functional pinealectomy" in our study was associated with increased heart weight similarly to 
A

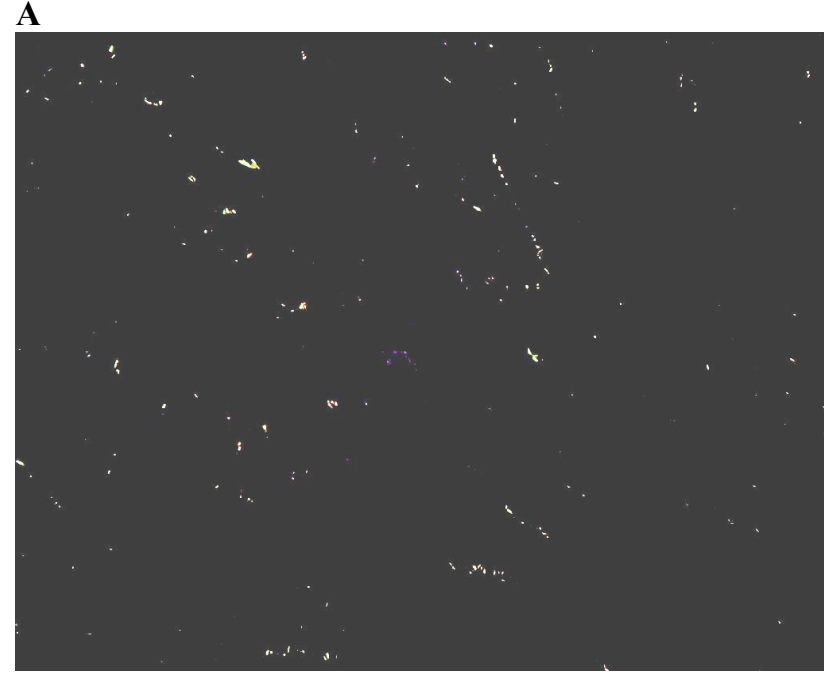

B

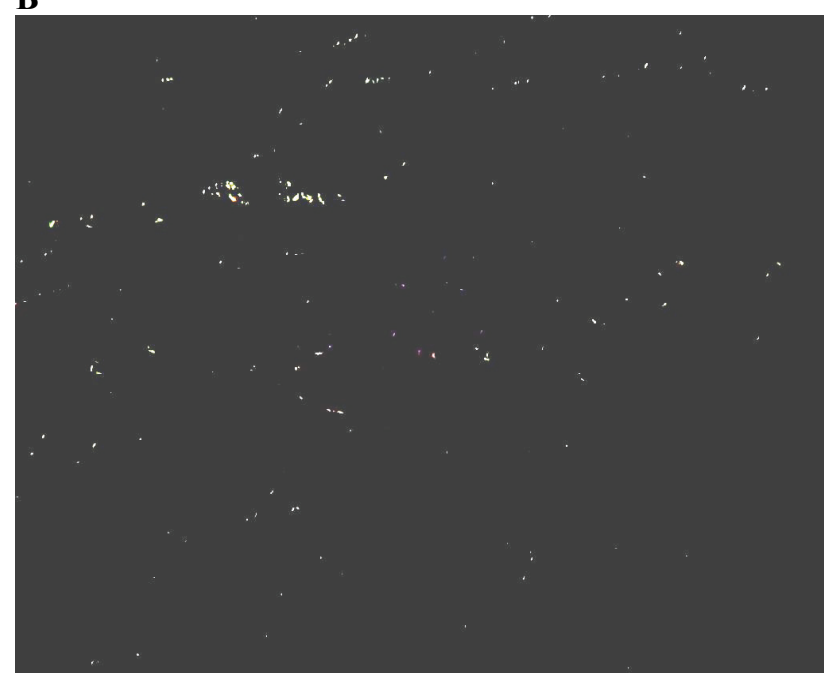

Fig. 3. Myocardial fibrosis analyzed in polarized light after picrosirius red staining in control group $(\mathbf{A})$ and rats exposed for 4 weeks to continuous $24 \mathrm{~h} /$ day light (B). The continuous light exposure (light) had no effect on fibrosis level. Final magnification 40x.

surgical pinealectomy (Mizrak et al. 2004). It was supposed that cardiac hypertrophy represented an adaptive response to increased hemodynamic load reported after pinealectomy (Mizrak et al. 2004). Nevertheless, the increased heart weight after surgical pinealectomy (Sahna et al. 2002a,b) as well as in our study using "functional pinealectomy" was proportional to the increase in body weight. Therefore the increase in cardiac mass is likely to be a result of body growth in conditions of increased food efficiency, insulin and leptin levels and decreased locomotor activity, which could be expected in melatonin-deficient animals (Wolden-Hanson et al. 2000). Moreover, the gain in heart weight after surgical pinealectomy did not correlate with blood pressure rise (Sahna et al. 2002a,b).

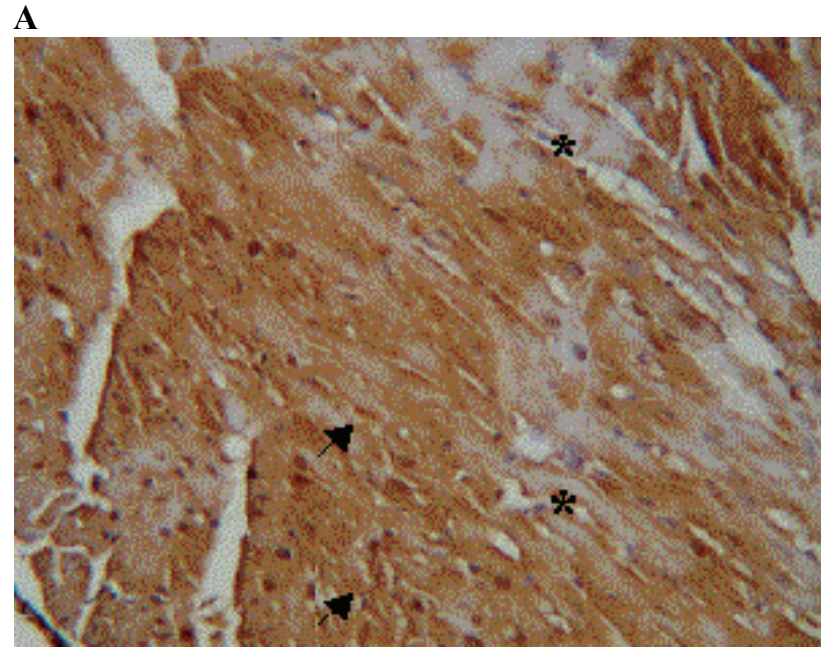

B

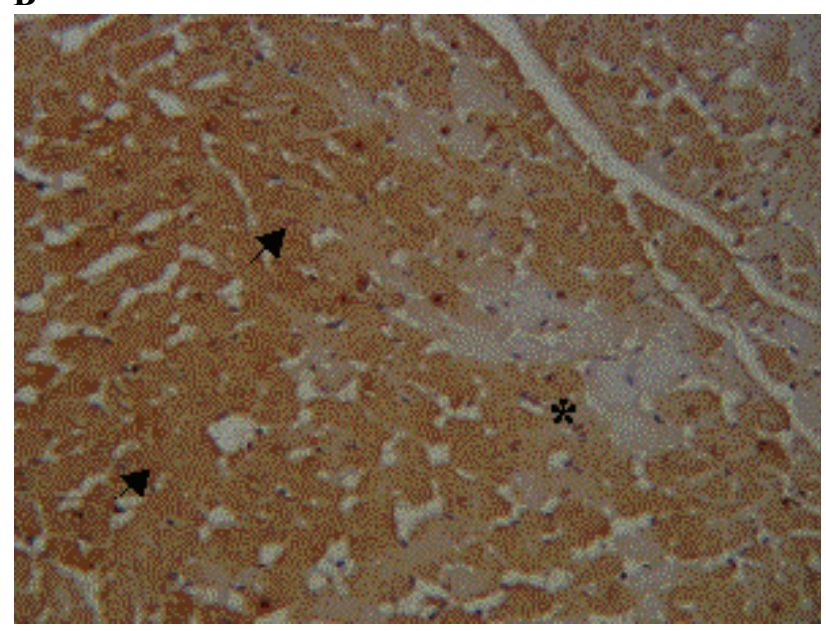

Fig. 4. Endothelial NO-synthase expression in control group (control) and rats exposed for 4 weeks to continuous $24 \mathrm{~h} /$ day light (light). One third of cardiomyocytes was not showing any NO-synthase positivity. There were no differences in the localization of NO-synthase positivity between the groups. Final magnification 40x, arrow, NO-synthase positivity, *, NO-synthase negativity.

In contrast to surgical pinealectomy (Mizrak et al. 2003), in this study the "functional pinealectomy" has not enhanced the level of myocardial fibrosis (Fig. 3). The absence of fibrosis after 4-week light exposure of rats might be explained by the fact that "functional pinealectomy" suppresses only night-time melatonin levels or that a longer period of continuous light is required to promote the development of myocardial fibrosis. Despite the unchanged level of fibrosis we found increased collagen I/III ratio. Type I and III collagens are major structural proteins forming the myocardial collagen matrix. Type I collagen determines the stiffness of the myocardium, while type III collagen contributes to its elasticity (Brilla et al. 1993, Yamamoto et al. 2002). It has been proposed that collagen type I/III ratio is an 
important marker for determination of the quality of collagen and prediction of myocardial stiffness (Pathak et al. 2001). The quality of collagen in the remodeling of myocardium might be responsible for adverse cardiovascular events (Weber 2000). Changes in collagen I/III ratio were also suggested to decrease electrical stability of myocardium (Xu et al. 2004) and could be involved in the modulation of the outcome of ischemiareperfusion in functionally/surgically pinealectomized rats. Excessive collagen content was proposed to be a result of increased collagen synthesis and/or inadequate collagen degradation (Varo et al. 2000). Both might be modulated by nitric oxide (Rossi et al. 2003). Attenuation of NO-synthase activity in the heart in association with continuous light exposure was observed in our previous recent experiments (Paulis et al. 2006).

The immunohistochemical study of NO-synthase expression was performed with the aim to elucidate whether previously reported changes in NO-synthase activity are caused by altered NO-synthase expression. We have not observed any changes in the localization or level of NO-synthase (either inducible or endothelial) positivity in cardiomyocytes (Fig. 4). However, reduced melatonin levels in light-exposed animals may result in attenuated antioxidant capacity of melatonin (Tan et al. 2000, Girouard et al. 2004, Sahna et al. 2005) and the lack of inhibitory effect of melatonin on glucocorti-coid formation (Rebuffat et al. 1987). Both these factors could inhibit NO formation (Maxwell 2002), without affecting the level of NO-synthase expression.

We conclude that four-week exposure of rats to continuous light has not affected relative heart weight, the level of myocardial fibrosis or NO-synthase expression but increased collagen type I/III ratio. The loss of structural homogeneity of the myocardium in favor of collagen I might increase myocardial stiffness and contribute to functional alterations after continuous light exposure.

\section{Acknowledgements}

This work was partially supported by the UK grant Nr. 29/2007, 30/2007, APVT grant 51-027404 and 51018004, VEGA grants 1/3429/06, 2/6148/26, 2/5110/25, and SP grant 51/0280900/0280901.

\section{References}

BARRETT T, KENT S, VOUDOURIS N: Does melatonin modulate beta-endorphin, corticosterone, and pain threshold? Life Sci 66: 467-476, 2000.

BRIAUD SA, ZHANG BL, SANNAJUST F: Continuous light exposure and sympathectomy suppress circadian rhythm of blood pressure in rats. J Cardiovasc Pharmacol Ther 9: 97-105, 2004.

BRILLA CG, REAMS GP, MAISCH B WEBER KT: Renin-angiotensin system and myocardial fibrosis in hypertension: regulation of the myocardial collagen matrix. Eur Heart J 14 (Suppl J): 57-61, 1993.

BROWN GM, BAR-OR A, GROSSI D, KASHUR S, JOHANNSON E, YIE SM: Urinary 6-sulphatoxylmelatonin, an index of pineal function in the rat. J Pineal Res 10: 141-147, 1991.

BRUGGER P, MARKTL W, HEROLD M: Impaired nocturnal secretion of melatonin in coronary heart disease. Lancet 345: 1408, 1995.

DELIBAS N, TUZMEN N, YONDEN Z, ALTUNTAS I: Effect of functional pinealectomy on hippocampal lipid peroxidation, antioxidant enzymes and N-methyl-D-aspartate receptor subunits $2 \mathrm{~A}$ and $2 \mathrm{~B}$ in young and old rats. Neuro Endocrinol Lett 23: 345-350, 2002.

DOLBER PC, SPACH MS: Conventional and confocal fluorescence microscopy of collagen fibers in the heart. J Histochem Cytochem 41: 465-469, 1993.

GARG UC, HASID A: Nitric oxide generating vasodilatators and 8-bromocyclic guanosine monophosphate inhibit mitogenesis and proliferation of cultured rat vascular smooth muscle cells. J Clin Invest 83: 1774-1777, 1989.

GIROUARD H, DENAULT C, CHULAK C, DE CHAMPLAIN J: Treatment by n-acetylcysteine and melatonin increases cardiac baroreflex and improves antioxidant reserve. Am J Hypertens 17: 947-954, 2004.

JONAS M, GARFINKEL D, ZISAPEL N, LAUDON M, GROSSMAN E: Impaired nocturnal melatonin secretion in non-dipper hypertensive patients. Blood Press 12: 19-24, 2003.

KOLPAKOV V, GORDON D, KULIK TJ: Nitric oxide-generating compounds inhibit total protein and collagen synthesis in cultured vascular smooth muscle cells. J Hypertens 9: 7-22, 1995. 
MAXWELL AJ: Mechanisms of dysfunction of the nitric oxide pathway in vascular diseases. Nitric Oxide 6: 101-124, 2002.

MIZRAK B, PARLAKPINAR H, ACET A, TURKOZ Y: Effects of pinealectomy and exogenous melatonin on rat hearts. Acta Histochem 106: 29-36, 2004.

PATHAK M, SARKAR S, VELLAICHAMY E, SEN S: Role of myocytes in myocardial collagen production. Hypertension 37: 833-840, 2001.

PAULIS L', JANEGA P, PECHÁŇOVÁ O, ZICHA J, HOJNÁ S, VAŽAN R: Continuous light exposure protects rat heart against ischemia-reperfusion injury. Physiol Res 55: 2P, 2006.

REBUFFAT P, MAZZOCCHI G, GOTTARDO G, COI A, MENEGHELLI V, NUSSDORFER GG: An ultrastructural morphometric study of the effects of chronic melatonin administration on the zona fasciculata of rat adrenal cortex. J Submicrosc Cytol 19: 415-421, 1987.

ROSSI MA, RAMOS SG, PRADO CM: Chronic inhibition of nitric oxide synthase induces hypertension and cardiomyocyte mitochondrial and myocardial collagen remodelling in the absence of hypertrophy. J Hypertens 21: 993-1001, 2003.

SAHNA E, OLMEZ E, ACET A: Effects of physiological and pharmacological concentrations of melatonin on ischemia-reperfusion arrhythmias in rats: can the incidence of sudden cardiac death be reduced? $J$ Pineal Res 32: 194-198, 2002a.

SAHNA E, ACET A, OZER MK, OLMEZ E: Myocardial ischemia-reperfusion in rats: reduction of infarct size by either supplemental physiological or pharmacological doses of melatonin. J Pineal Res 33: 234-238, 2002b.

SAHNA E, PARLAKPINAR H, TURKOZ Y, ACET A: Protective effects of melatonin on myocardial ischemia/reperfusion induced infarct size and oxidative changes. Physiol Res 54: 491-495, 2005.

TAN DX, MANCHESTER LC, REITER RJ, QI WB, KARBOWNIK M, CALVO JR: Significance of melatonin in antioxidative defense system: reactions and products. Biol Signals Recept 9: 137-159, 2000.

VARO N, IRABURU MJ, VARELA M, LOPEZ B, ETAYO JC, DIEZ J: Chronic $\mathrm{AT}_{1}$ blockade stimulates extracellular collagen type I degradation and reverses myocardial fibrosis in spontaneously hypertensive rats. Hypertension 35: 1197-1202, 2000.

VAŽAN R, BÉDER I, STYK J: Melatonin and the heart (in Slovak). Cesk Fysiol 53: 29-33, 2004.

WEBER KT: Targeting pathological remodeling: concepts of cardioprotection and reparation. Circulation 102: 1342$1345,2000$.

WITTE K, GREBMER W, SCALBERT E, DELAGRANGE P, GUARDIOLA-LEMAITRE B, LEMMER B: Effects of melatoninergic agonists on light-suppressed circadian rhythms in rats. Physiol Behav 65: 219-224, 1998.

WOLDEN-HANSON T, MITTON DR, MCCANTS RL, YELLON SM, WILKINSON CW, MATSUMOTO AM, RASMUSSEN DD: Daily melatonin administration to middle-aged male rats suppresses body weight, intraabdominal adiposity, and plasma leptin and insulin independent of food intake and total body fat. Endocrinology 141: 487-497, 2000.

XU J, CUI G, ESMAILIAN F, PLUNKETT M, MARELli D, ARDEHALI A, ODIM J, LAKS H, SEN L: Atrial extracellular matrix remodeling and the maintenance of atrial fibrillation. Circulation 109: 363-368, 2004.

YAMAMOTO K, MASUYAMA T, SAKATA Y, NISHIKAWA N, MANO T, YOSHIDA J, MIWA T, SUGAWARA M, YAMAGUCHI Y, OOKAWARA T, SUZUKI K, HORI M: Myocardial stiffness is determined by ventricular fibrosis, but not by compensatory or excessive hypertrophy in hypertensive heart. Cardiovasc Res 55: 76-82, 2002.

ZANOBONI A, ZANOBONI-MUCIACCIA W: Experimental hypertension in pinealectomized rats. Life Sci 6: 23272331, 1967.

\section{Corresponding author}

L. Paulis, Institute of Pathophysiology, School of Medicine, Comenius University, Sasinkova 4, 81108 Bratislava, Slovak Republic. Fax: +421-(0)2-59357601. E-mail: ludo@lfuk.sk 